\title{
Boron quantum dots all-optical modulator based on efficient photothermal effect
}

\author{
Cong Wang ${ }^{1 \dagger}$, Qianyuan Chen ${ }^{2 \dagger}$, Hualong Chen ${ }^{1}$, Jun Liu ${ }^{1}$, Yufeng Song ${ }^{1}$, \\ Jie $\mathrm{Liu}^{3}$, Delong $\mathrm{Li}^{1}$, Yanqi $\mathrm{Ge}^{1}$, Youning Gong ${ }^{1 *}$, Yupeng Zhang ${ }^{1 *}$ and \\ Han Zhang ${ }^{1 *}$
}

All-optical devices without external electronic components have drawn extraordinary attentions in all-optical communication. In this work, boron quantum dots (BQDs) were synthesized by a facile liquid-phase exfoliation method. The as-prepared BQDs showed good structural homogeneity and crystallinity, broadband optical absorption as well as excellent photothermal properties. Femtosecond-resolved transient absorption further revealed the short carrier relaxation time of BQDs. Inspired by the outstanding photothermal properties and ultrafast carrier dynamic of BQDs, we fabricated BQDsbased all-optical modulator. The phase shift with a slope efficiency of $0.032 \mathrm{~m} / \mathrm{mW}$ and response time of $0.97 \mathrm{~ms}$ can be achieved. The modulator was used in laser resonance cavity to achieve all-optical actively Q-switched laser operation with control repetition rate. This prototypical BQDs-based all-optical modulator shows a great potential to be applied in all-optical information processing and communication.

Keywords: boron quantum dots; all-optical modulator; photothermal conversion; actively Q-switched laser

Wang C, Chen QY, Chen HL, Liu J, Song YF et al. Boron quantum dots all-optical modulator based on efficient photothermal effect. Opto-Electron Adv 4, 200032 (2021).

\section{Introduction}

Nanosecond lasers have been widely applied in many fields including laser manufacturing and etching, spectroscopy, and nonlinear optics due to their low cost and high output of energy ${ }^{1}$. Both passive and active Qswitched techniques are effective strategies to generate the nanosecond laser. For passively Q-switched method, the pulse laser operation is typically induced by the saturable absorber such as the semiconductor saturable ab- sorption mirror, carbon nanotubes, low-dimensional materials owing to their saturable absorption effect and ultrafast optical response ${ }^{2-6}$. For saturable absorberbased passive Q-switching regime, these materials may experience unstable optical performance resulting from oxidation and damage under illumination of the strong light, so the output pulse laser encounter challenges in achieving precisely controllable repetition rates. These unsolved problems render the practical application of

${ }^{1}$ Collaborative Innovation Center for Optoelectronic Science \& Technology, International Collaborative Laboratory of 2D Materials for Optoelectronics Science and Technology of Ministry of Education, Institute of Microscale Optoelectronics, Shenzhen University, Shenzhen 518060, China; ${ }^{2}$ School of Physics and Technology, and MOE Key Laboratory of Artificial Micro-and Nano-Structures, Wuhan University, Wuhan 430072, China; ${ }^{3}$ Shandong Provincial Engineering and Technical Center of Light Manipulation \& Shandong Provincial Key Laboratory of Optics and Photonic Device, School of Physics and Electronics, Shandong Normal University, Jinan 250014, China.

tThese authors contributed equally to this work.

"Correspondence: YN Gong, E-mail: youninggong@szu.edu.cn; YP Zhang, E-mail: ypzhang@szu.edu.cn; H Zhang, E-mail: hzhang@szu.edu.cn Received: 16 April 2020; Accepted: 9 October 2020; Published: 25 July 2021

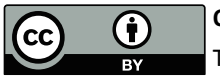

Open Access This article is licensed under a Creative Commons Attribution 4.0 International License.

C The Author(s) 2021. Published by Institute of Optics and Electronics, Chinese Academy of Sciences. 
passively Q-switched technology. Comparing with the passive counterpart, active Q-switched technique can achieve flexible change of repetition rate by adjusting the external driven Q-switcher, which have a significant effect on the transmission of optical information. Traditionally, taking advantage of electro-optical or acousticoptical modulator can achieve actively Q-switched laser generation, which are complicated, with narrowband optical responses, and difficult in all-optical operation. Recently, all-optical modulator (AOM) has attracted intense interests due to their low energy loss, broadband modulation, and compatibility and integration with alloptical network ${ }^{7-10}$. Various physical mechanisms applied into AOM have been developed to realize actively Q-switched laser, including graphene-based cross absorption modulation ${ }^{11}$, resonant optical pumping of fiber Bragg grating ${ }^{12}$ and modulation of rare-earth-doped fiber saturable absorber ${ }^{13}$. However, these strategies still have some restrictions involving specific wavelength operation and low modulation depth. AOM using the photothermal effect of materials shows a good possibility to overcome these issues for its excellent properties such as large modulation depth and easy implementation. So far, various low-dimensional nanomaterials including graphene $^{14-17}$, transition metal dichalcogenides ${ }^{9,18}$, phosphorene ${ }^{19}$, black phosphorus analogues (tellurene, bismuthine, and antimonene) $)^{20,21}$ and MXene ${ }^{22}$ have been widely applied to AOM because of their excellent optical absorption properties and strong photothermal effect. Continuous exploration of new materials is conducive to the improvement of modulator's performance.

As the neighbor of carbon in the periodic table, boron is one of the most chemically versatile elements. Due to the trivalent electronic configuration of boron, $\mathrm{B}-\mathrm{B}$ bonds tend to form the highly delocalized multicenter bonding, which leads to the polymorphism of boron allotropes in different dimensions, such as boronphene, boron nanotubes, and boron quantum dots (BQDs) ${ }^{23}$. Low-dimensional boron nanomaterials have been long concerned by theoretical researchers ${ }^{24-27}$. For example, the boronphene possesses many intriguing properties such as high carrier concentration and mobility, phonon-mediated superconductivity, visible/near-infrared plasmons ${ }^{24,25,28}$. Ultra-small sized quantum dots known as "artificial atoms" can be regarded as the enhancer due to their strong quantum confinement effect and high extinction coefficients ${ }^{29}$. However, experimental researches about BQDs are relatively rare, because the chemical and structural complexities pose severe challenges to the synthesis of low-dimensional boron nanomaterials. Therefore, the synthesis of BQDs is of great significance for the research of their thermal and optical properties.

In this contribution, boron quantum dots (BQDs) were synthesized via a facile liquid-phase exfoliation process. The BQDs exhibited an excellent optical absorption property and photothermal effect. They also possessed fast relaxation time with $194 \mathrm{fs}$ and 15.1 ps at $970 \mathrm{~nm}$, which are much shorter than those of other state-of-theart nanomaterials such as graphene, $\mathrm{WS}_{2}$ and black phosphorus. This makes BQDs a promising candidate to be applied in the ultrafast photonics and high-speed optical switcher. For the all-optical modulator, the phase shift with a slope efficiency of $0.032 \pi / \mathrm{mW}$ and response time of $0.97 \mathrm{~ms}$ is superior to similar devices based on other state-of the-art 2D materials. Moreover, for the actively Q-switched laser, the output pulse laser achieved controlled repetition rate arranged from $1.2 \mathrm{kHz}$ to 2.5 $\mathrm{kHz}$ utilizing the proposed all-optical modulator. This work may boost the experimental study of low-dimensional boron nanomaterials, as well as the research on its optical properties and related applications.

\section{Characterization of BQDs}

The probe ultrasonication and high energy ball milling were combined to exfoliate bulk boron in liquid media to obtain BQDs. First, bulk boron powder with an average lateral particle size of $\sim 10 \mu \mathrm{m}$ was dispersed into dimethylformamide (DMF), followed by ultrasonication to induce initial exfoliation. The resultant sample was transferred into a ball milling jar to proceed further mechanochemical reactions to produce BQDs. As shown in Figs. 1(a) and 1(b), the atomic force microscopy (AFM) image and transmission electron microscopy (TEM) micrograph reveal the relatively uniform size of BQDs. The average diameter is $7.1 \mathrm{~nm}$, calculated from the statistical diameter distribution (Inset of Fig. 1(a)). The crystalline nature of BQDs was demonstrated by HRTEM, in which clear interference fringes with a dspacing of $0.50 \mathrm{~nm}$ were observed, corresponding to the (104) plane of $\beta$-rhombohedral boron structure ${ }^{30}$. The elemental compositions were determined by electron energy loss spectroscopy (EELS) and X-ray photoelectron spectroscopy (XPS). Figure 1(c) displays the EELS spectrum of BQDs, which shows a characteristic boron Kshell ionization edge at $\sim 188 \mathrm{eV}$. The corresponding elemental contents of $\mathrm{B}, \mathrm{C}$ and $\mathrm{O}$ were extracted from EELS 
spectrum, which are 60.29 at. \%, 36.44 at. \%, 3.28 at. \%, respectively. The existence of $\mathrm{C}$ and $\mathrm{O}$ is mainly caused by the surface contamination from ambient atmosphere and organic solvent, which is further confirmed by XPS measurement. From the high-resolution B 1s XPS spectrum in Fig. 1(d), three peaks at 187.6, 189.3 and 191.3 $\mathrm{eV}$ can be resolved, corresponding to three bonding types of boron. The main peak at $187.6 \mathrm{eV}$ is assigned to $\mathrm{B}-\mathrm{B}$ bonds, which is consistent with the reported value obtained from bulk boron (187.3-187.9 eV) ${ }^{31,32}$. Peaks with higher binding energy at 189.3 and $191.3 \mathrm{eV}$ are corresponded to the oxidation state of boron, wherein $189.3 \mathrm{eV}$ is assigned to $\mathrm{B}-\mathrm{O}$ bonds and $191.3 \mathrm{eV}$ to $\mathrm{B}-\mathrm{C}$ bonds. The very weak intensity of these two peaks reveals the good stability of BQDs against oxidation. Figure 1 (e) provides the optical absorption of BQDs from 200 to $1600 \mathrm{~nm}$, while a broad spectral absorption can be seen in the spectrum, especially in the visible region. It is noteworthy that BQDs shows the enhanced absorption, when compared to the $2 \mathrm{D}$ boron nanosheets reported previous literature ${ }^{33}$.

Inspired by the intriguing structural and optical properties, we conducted the photothermal experiments on BQDs. In experiment, a microfiber with a waist diameter of about $7 \mu \mathrm{m}$ was fabricated by tapering a standard single-mode fiber by flame heating. When a $980 \mathrm{~nm}$ laser was guided into the fabricated microfiber, the prepared BQDs dropped onto the microfiber was absorbed on the surface of the microfiber due to optical gradient force (Inset of Fig. 1(f)), resulting in the absorption loss of 3.5 $\mathrm{dB}$ at $980 \mathrm{~nm}$. The evanescent field of microfiber interacted with BQDs to generate Joule heat to change the refractive index of nanomaterials. The temperature of the BQDs-deposited microfiber was monitored by a thermal a
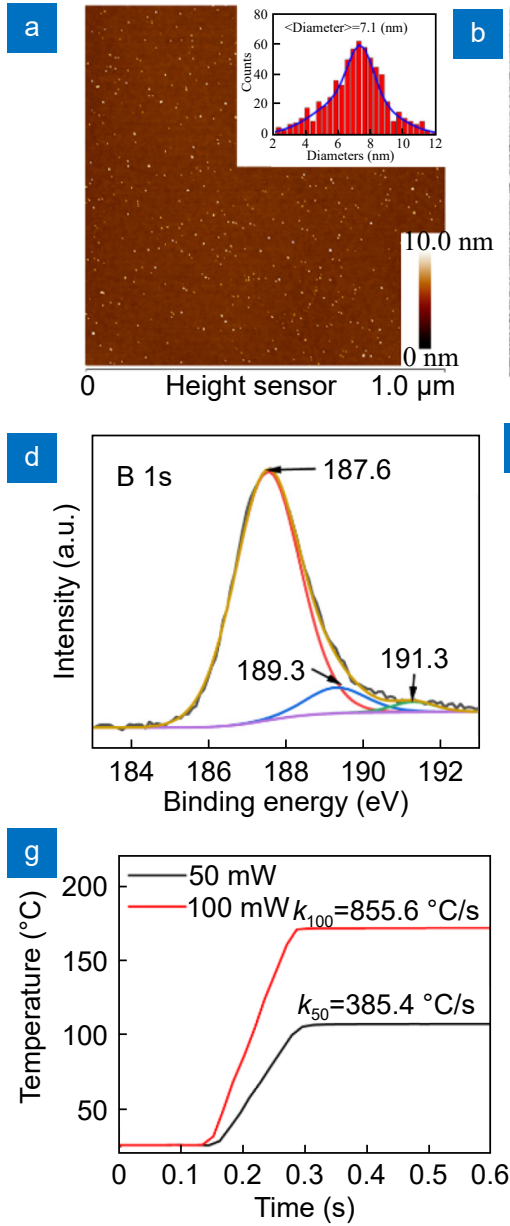
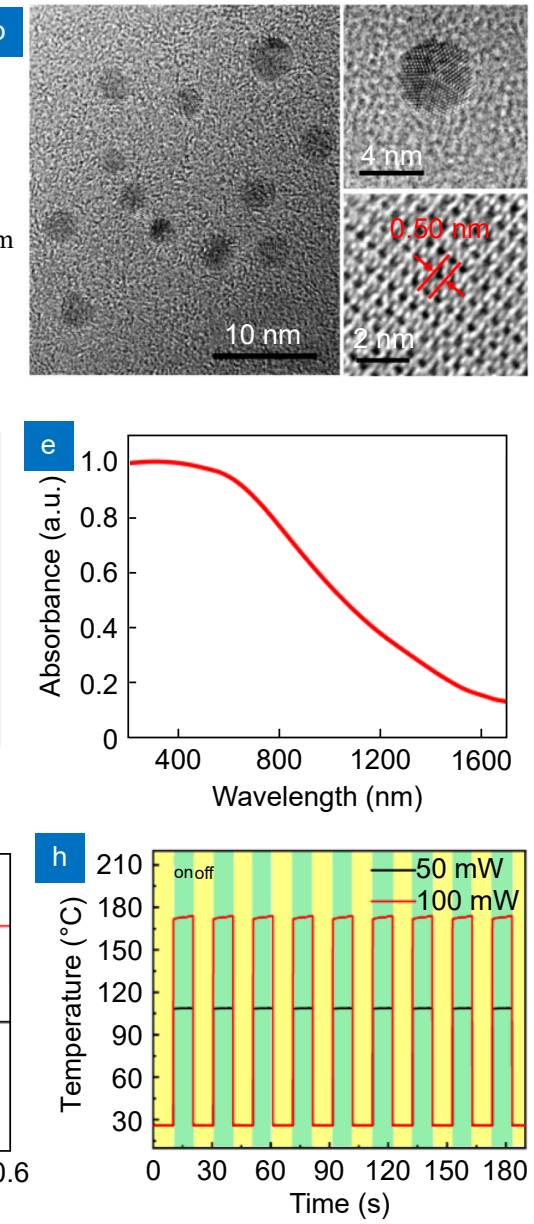

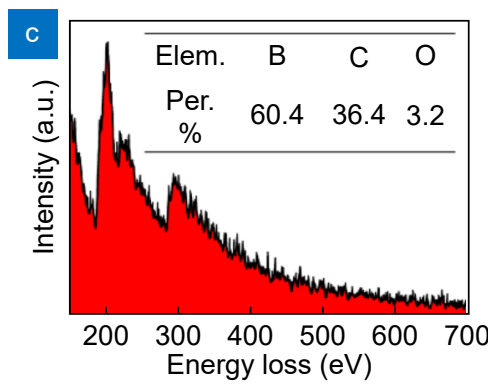

f
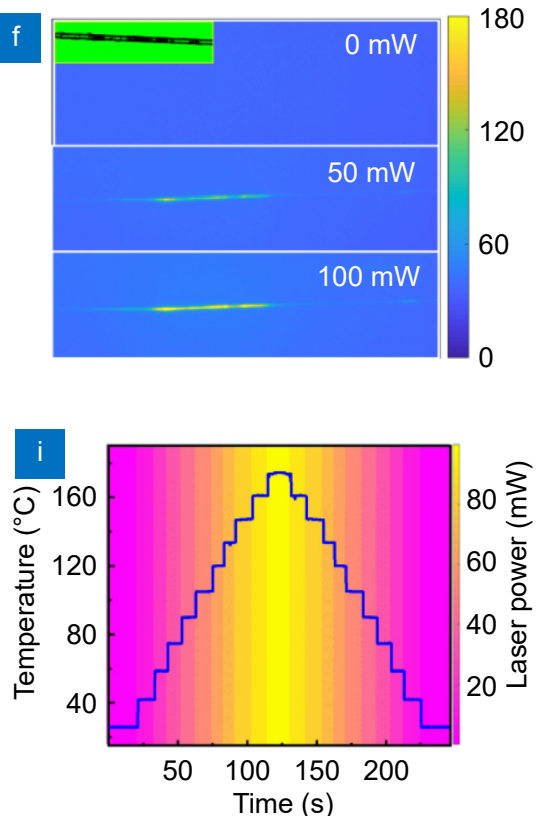

Fig. 1 | Characterization and photothermal properties of BQDs. (a) AFM image with statistical data of diameters (inset). (b) HRTEM micrographs. (c) EELS spectrum with the corresponding atomic ratio. (d) XPS spectrum of B 1s. (e) UV-Vis-NIR absorption spectrum. (f) IR thermogram of the BQDs-deposited microfiber with the incident light power of $0 \mathrm{~mW}, 50 \mathrm{~mW}$ and $100 \mathrm{~mW}$, respectively, inset shows the optical microscopic image of BQDs-deposited microfiber. (g) Photothermal response rate at $50 \mathrm{~mW}$ and $100 \mathrm{~mW}$. (h) Photothermal switching performance at $50 \mathrm{~mW}$ and $100 \mathrm{~mW}$. (i) Temperature variation with light power from 0 to $100 \mathrm{~mW}$ with an interval of $10 \mathrm{~mW}$. 
imaging camera (Optotherm IS640). The temperature showed distinct variation under different incident light power from 0 to $100 \mathrm{~mW}$ (Fig. 1(f)), which indicates the efficient photothermal conversion of BQDs. Figure 1(g) shows photothermal heating curves of BQDs varies with time at 50 and $100 \mathrm{~mW}$. BQDs showed a very rapid heating rate of 385.4 and $855.6^{\circ} \mathrm{C} / \mathrm{s}$ at 50 and $100 \mathrm{~mW}$, respectively. Moreover, the highest temperature can be reached $175{ }^{\circ} \mathrm{C}$ at the light power of $100 \mathrm{~mW}$. Figure 1 (h) displays the switching test under the light power of 50 and $100 \mathrm{~mW}$. No degradation was observed for the heating rate or temperature, indicating the excellent thermal stability of BQDs. As shown in Fig. 1(i), with the light power gradually increasing from $0 \mathrm{~mW}$ to $100 \mathrm{~mW}$ at a step of $10 \mathrm{~mW}$ and then recovered to $0 \mathrm{~mW}$, the temperature of microfiber varied with uniform and high symmetry, indicating the excellent thermal recovery of BQDs. The excellent characteristics of BQDs including fast response speed, stable energy converison and outstanding thermal recovery properties laid the foundation for achieving high-performance photonics devices.

To gain a deeper insight about the optical characteristics of BQDs, a femtosecond-resolved transient absorption (TA) spectrometer is applied to study the carrier relaxation behaviors. The photo-excited carriers dynamic in nanomaterials has a significant impact on optoelectronic and photonic devices, which plays a guiding role on light-matter interaction and interfacial engineering between nanomaterials and substrate. A femtosecond-resolved transient absorption (TA) spectrometer is applied to study the carrier relaxation behaviors of BQDs. In our experiment, the detailed information of TA spectrometer is provided in Figure S1 of Supplementary Information, the ultrafast laser at $400 \mathrm{~nm}$ acted as pump light stimulates electrons of ground state to excited state. The laser at $500-730 \mathrm{~nm}$ and $910-1070 \mathrm{~nm}$ is regarded as the probe light. The $2 \mathrm{D}$ mapping TA spectra of BQDs includes temporally and spectrally resolved TA signal, as shown in Fig. 2(a) and 2(b), suggesting broadband ground state bleaching transient response and ultrafast carrier process within the range of picoseconds response. In Fig. 2(c) and 2(d), we obtain two dynamic curves (520 $\mathrm{nm}$ and $970 \mathrm{~nm}$ ) of the BQDs to analyse the relaxation process. These curves at different wavelengths illustrate ground state bleaching properties and fast response, which can be well fitted by a biexponential function $\Delta A=A_{1} \exp \left(-t / \tau_{1}\right)+A_{2} \exp \left(-t / \tau_{2}\right)$, where $A_{1}$ and $A_{2}$ are the amplitudes, $t$ is the delay time between pump light and probe light, $\tau_{1}$ and $\tau_{2}$ represent the time constants corresponding to the carrier lifetime of BQDs. The time constants $\left(\tau_{1}\right.$ and $\left.\tau_{2}\right)$ are closely related to the cooling of hot excitons and recombination processes of excitons in BQDs, corresponding to electron-phonon interaction and phonon-phonon interaction. Obviously, the BQDs possess picosecond relaxation time with $\tau_{1}$ of $771 \mathrm{fs}$ and $\tau_{2}$ of $6.8 \mathrm{ps}$ at $520 \mathrm{~nm}, \tau_{1}$ of $194 \mathrm{fs}$ and $\tau_{2}$ of $15.1 \mathrm{ps}$ at 970 $\mathrm{nm}$, establishing a good basis to achieve high-performance optoelectronic devices. In Table S1 of Supplementary Information, the carrier dynamic of different nanomaterials are summarized. Relaxation time constants of BQDs are much shorter than those of other nanomaterials such as graphene, $\mathrm{WS}_{2}$ and black phosphorus ${ }^{34-38}$. The ultrafast carrier dynamic of BQDs may have the potentials to achieve breakthrough in ultrafast photonics and high-speed optical switcher.

\section{All-optical phase and intensity modulator}

In experiment, a BQDs-deposited microfiber with a diameter of $6 \mu \mathrm{m}$ and a length of $39 \mu \mathrm{m}$ was placed in the all-optical modulator (Fig. 3(a)). The all-optical modulator was made up of a Michelson interferometer (MI) including two light sources (control light and signal light) and two arms (reference arm and modulation arm). The signal light with a center wavelength of 1550 $\mathrm{nm}$ and the control light with a center wavelength of 980 $\mathrm{nm}$ were coupled into the modulator by a wavelength division multiplexer (WDM), and injected into two arms by an optical coupler (OC) with a splitting ratio about $50: 50$ at $1550 \mathrm{~nm}$ and $90: 10$ at $980 \mathrm{~nm}$, respectively. The $90 \%$ control light was transmitted in the modulation arm and interacted with BQDs, and the generated heat will change the refractive index of materials and fiber to modulate the phase between two arms. The power of the $10 \%$ one was controlled by the variable optical attenuators (VOA) in reference arm. Faraday rotation mirror was used to reflect the signal light. The reflected signal light interfered with each other at OC and partly exported at output port. The output signal light was analyzed by an optical spectrum analyzer (Yokokawa, AQ6370D).

At first, an amplified spontaneous emission (ASE) light (the bandwidth of $40 \mathrm{~nm}$ ) acted as the signal light was injected into the MI structure. By adjusting the VOA, the power difference of two arms is almost equal, which is beneficial to achieve maximum interference contrast (Fig. 3(b)). As shown in Fig. 3(c), we obtain 

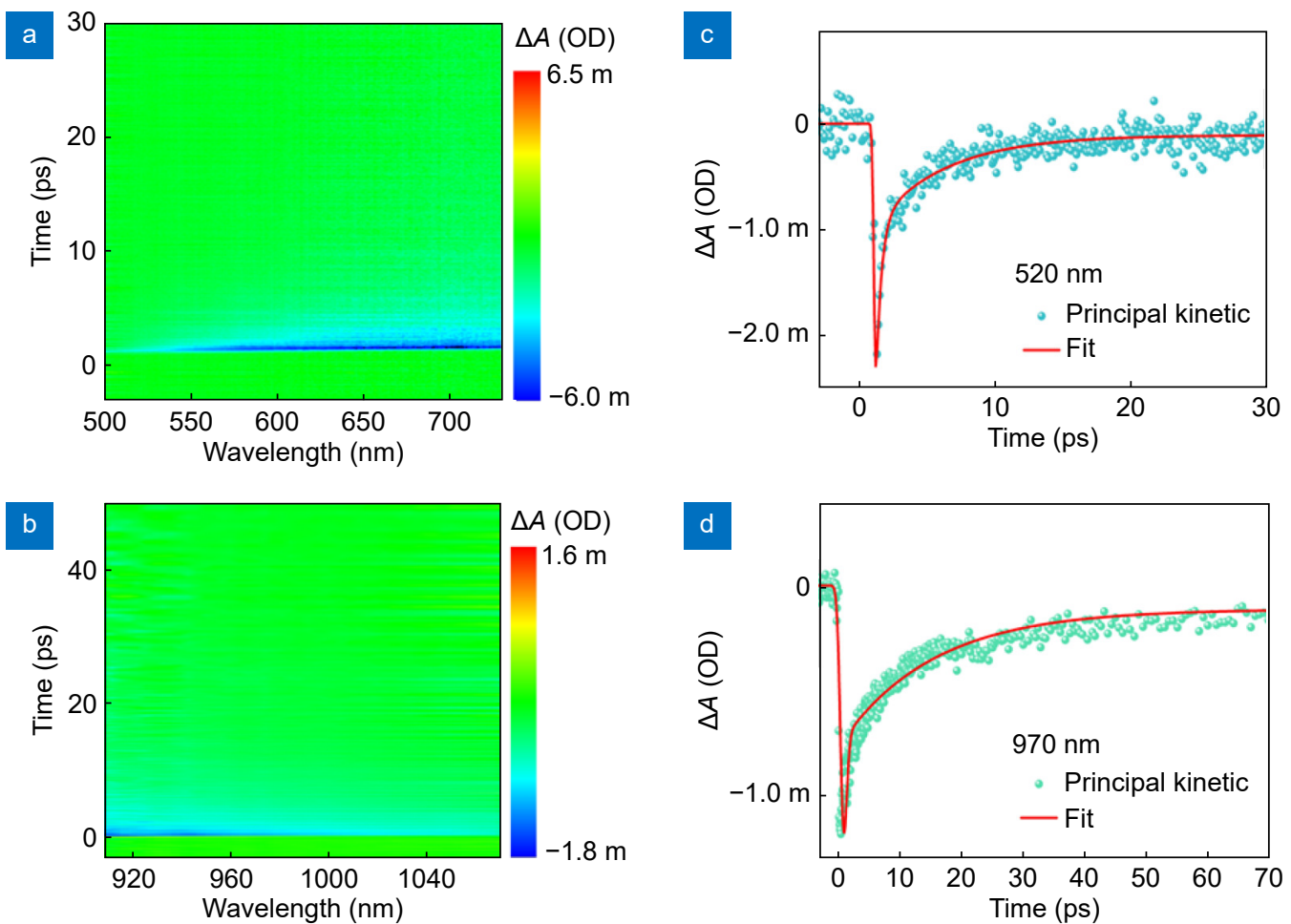

Fig. 2 | (a, b) 2D mapping of TA spectra from $500 \mathrm{~nm}$ to $730 \mathrm{~nm}$, and from $910 \mathrm{~nm}$ to $1070 \mathrm{~nm}$. Horizontal axis, vertical axis, and color scale represent the probe wavelength, the pump-probe time delay, and the intensity of TA signal, respectively. The dynamic curves and fitted results of the BQDs at $520 \mathrm{~nm}(\mathbf{c})$ and $970 \mathrm{~nm}(\mathbf{d})$.

interferometric spectrum with a free spectral range (FSR) of $0.6 \mathrm{~nm}$ and a modulation depth of $24.5 \mathrm{~dB}$, indicating the optical path difference of two arms is $1.4 \mathrm{~mm}$. When a $980 \mathrm{~nm}$ laser is guiding into the all-optical modulator, BQDs absorb the control light to change the phase difference between two arms of MI structure, resulting in the interferometric spectrum of signal light shifting toward long wavelength. In Fig. 3(d), the spectrum shifts about $0.3 \mathrm{~nm}$ at the control light power of $33 \mathrm{~mW}$. When decreasing the control light to $0 \mathrm{~mW}$, the interferometric spectrum goes back to initial position in an opposite direction. In addition, we observe that the modulation depth and FSR of the interferometric spectrum have not been influenced by the control light power. In the process of increasing control light power, the spectrum variation is recorded. Figure 3(e) shows that the phase variation is linear with a slope of $0.032 \pi / \mathrm{mW}$ as a function of control light power, corresponding to the slope of 0.01 $\mathrm{nm} / \mathrm{mW}$. The maximum phase shift of $10 \pi$ is achieved at the control light power of $315 \mathrm{~mW}$.

From the interferometric spectrum in Fig. 3(b), the MI-based all-optical modulator is equivalent to an optically-controlled tunable comb filter, which can be described by a cosine function, $T=\left(1+\cos \left(\Delta \varphi_{0}+\Delta \varphi_{1}\right)\right) / 2$, where $T$ is the transmittance, $\Delta \varphi_{0}$ and $\Delta \varphi_{1}$ are the im- mobile phase difference induced by length difference of two arms and dynamic phase difference in connection with refractive index variation. When guiding a signal light with good monochromaticity into the MI structure, the output signal light will possess high transmittance if the center wavelength of the signal light is located near the peak wavelength of the transmission curve. On the contrary, the output intensity of signal light will be at a low level. As shown in Fig. 3(d), with the moving of interferometric spectrum, the intensity of a narrow-band signal light will be modulated by the external control light light to achieve all-optical intensity modulation. Therefore, the broadband ASE signal light is replaced by a distributed feedback laser with a bandwidth of $0.1 \mathrm{~nm}$ and a center wavelength of $1550 \mathrm{~nm}$.

In Fig. 4(a), when a square-wave control light with a modulation frequency of $300 \mathrm{~Hz}$ and optical power of 96 $\mathrm{mW}$ is coupled into the MI structure, the signal light loads and copies the information from control light with the same frequency and duty cycle (Fig. 4(b)). The waveform of signal light is edge-smoothed square wave with the frequency of $300 \mathrm{~Hz}$. In Fig. 4(c), the smooth edges of signal light are fitting by the exponential functions $1-\exp \left(-t / t_{\mathrm{r}}\right)$ and $\exp \left(-t / \mathrm{t}_{\mathrm{f}}\right)$, the time constants $t_{\mathrm{r}}$ and $t_{\mathrm{f}}$ are $0.5 \mathrm{~ms}$ and $0.6 \mathrm{~ms}$ for rising edge and falling edge, 

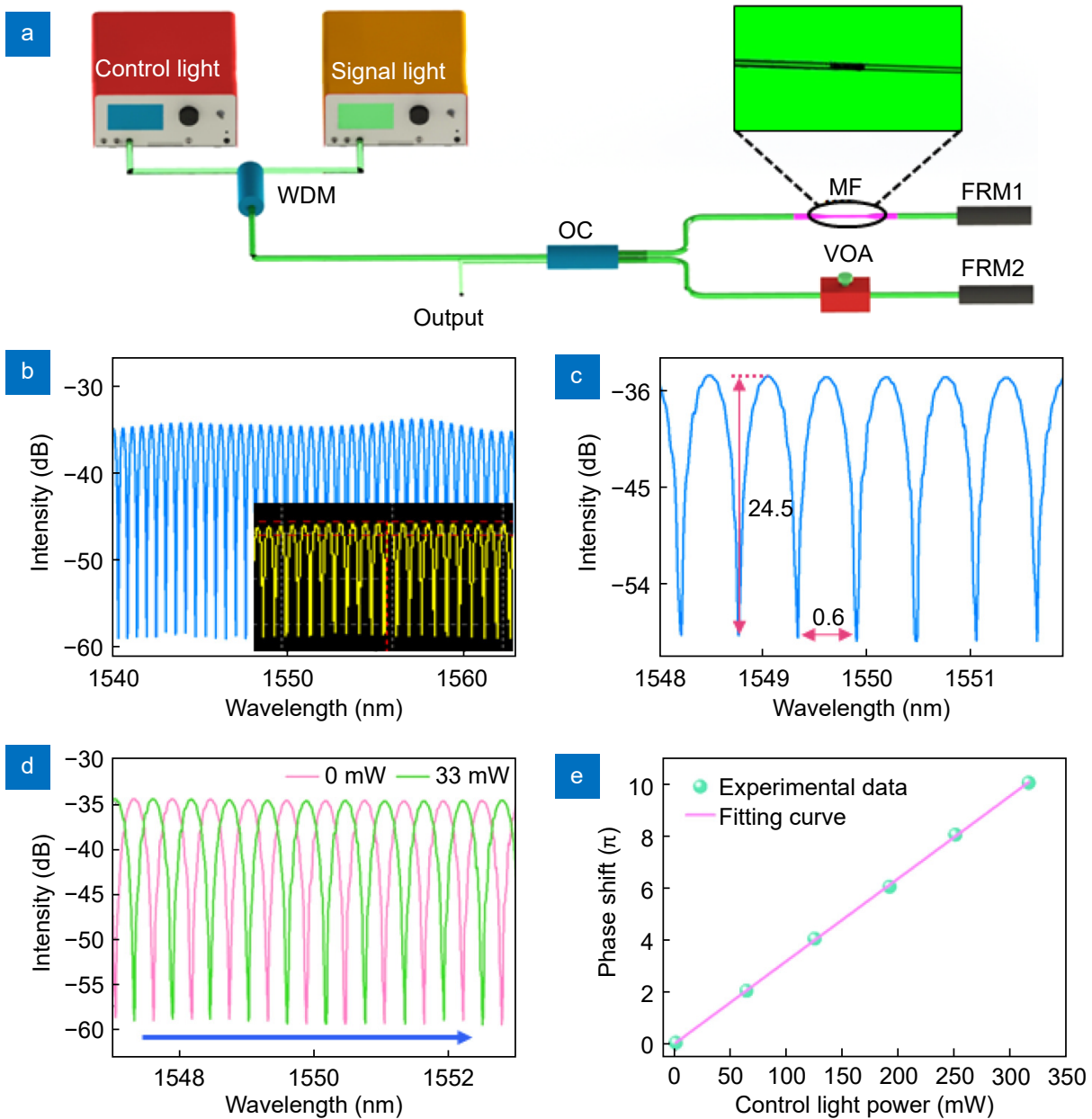

Fig. 3 | (a) The experimental diagram of the all-optical modulator. (b, c) Interferometric spectrum of signal light. (d) Interferometric spectra at the control light power of $0 \mathrm{~mW}$ and $33 \mathrm{~mW}$. (e) Phase shift versus the control light power.
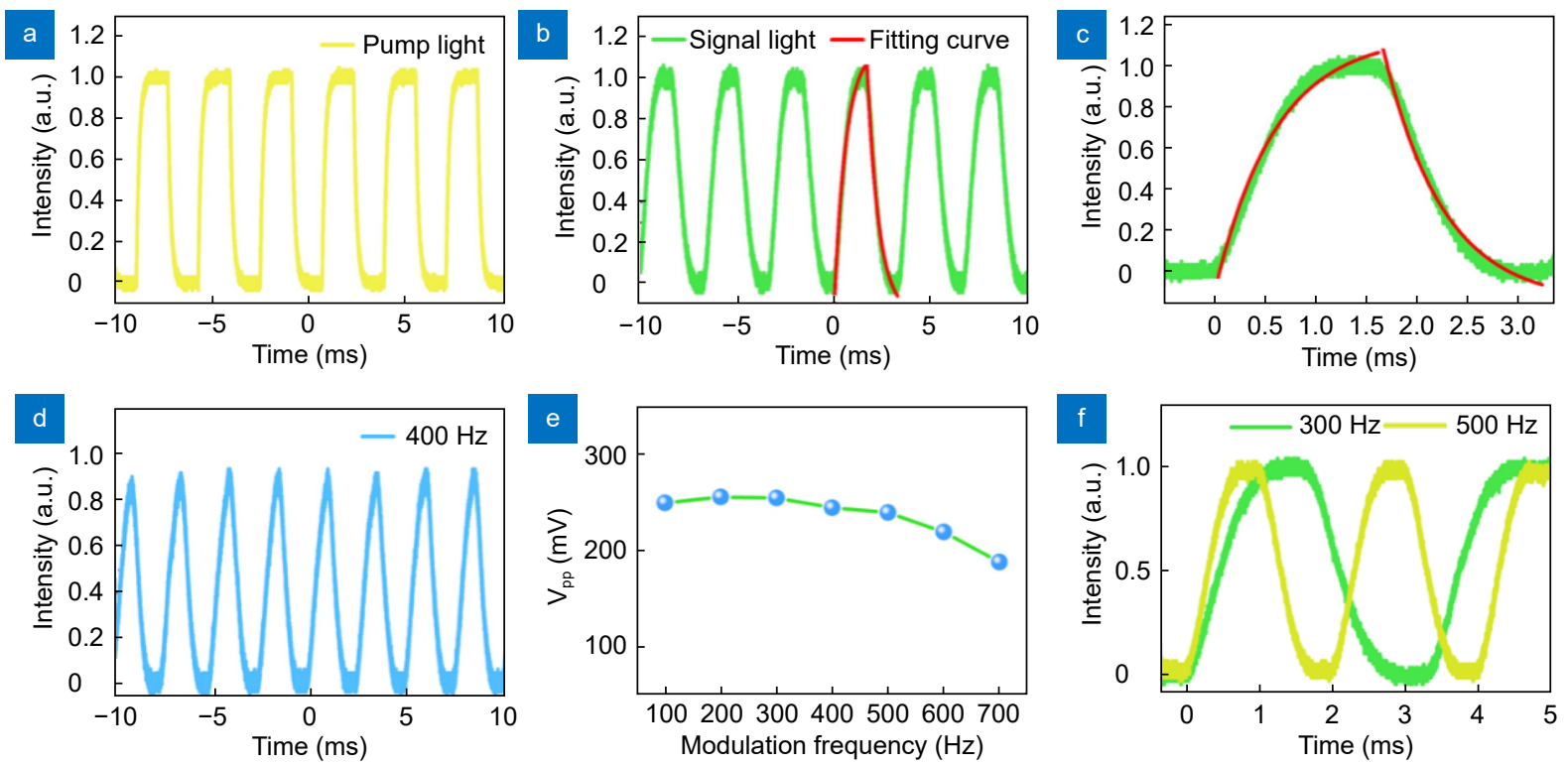

Fig. 4 | All-optical intensity modulation. (a, b) Waveform of control light and signal light at the modulation frequency of $300 \mathrm{~Hz}$. (c) A single on-off transition of the signal light and corresponding fitting curve. (d) Waveform of signal light at the modulation frequency of $400 \mathrm{~Hz}$. (e) $\mathrm{V}_{\mathrm{pp}}$ versus modulation frequency. (f) Waveform of signal light. 
corresponding to the rising time of $1.1 \mathrm{~ms}$ and the falling time of $1.3 \mathrm{~ms}$. To our knowledge, the response time is faster than the others similar devices, resulting from the superior photothermal response of BQDs. Low mass of boron atom and high stiffness of lattice structure cause higher electron-phonon coupling and higher phonon velocities, causing more efficient thermal generation and transport. In Table 1, we summarized the time response of current state-of-the-art fiber-typed all-optical modulator. It is clearly that BQDs-based all-optical modulator exhibits shorter rising time and falling time compared with similar optical structure, which results from more efficient thermal generation and dissipation. Due to the photothermal response of BQDs, the proposed all-optical modulator is limited to be $\sim \mathrm{kHz}$, which can be applied in some fields without the demand of high modulation speed. By using thinner microfiber, smaller footprint waveguide, and thinner nanomaterials with fast heat generation and diffusion, $\mathrm{AOM}$ will have faster response time as shown in Table 1. Micro-ring resonator optical structure is an effective way to achieve high-speed optical modulator. To achieve high-speed information processing, the ultrafast carrier dynamic process of BQDs measured by pump-probe setup (fs ps) supports the design of ultrahigh-speed optical modulators with a modulation bandwidth of $1000 \mathrm{GHz}$.

With the frequency of control light increases, not only the waveform of signal light is gradually distorted from edge-smoothed square wave to sharp triangular wave (Fig. 4(d)), but also the peak-to-peak voltage $\left(\mathrm{V}_{\mathrm{pp}}\right)$ of the signal light decreases form $250 \mathrm{mV}$ to $189 \mathrm{mV}$ (Fig. 4(e)). The reason for the phenomenon is that the response time of the thermo-optic effect is hard to keep up with the large modulation frequency, resulting in a challenge to achieve greater phase shift. In Fig. 4(f), we investigate the relationship among response time of signal light, the modulation frequency and optical power of control light in order to achieve square wave output. The control light with great modulation frequency and high optical power is easier to obtain fast-response signal light, which results from the greater phase generation under high control light power. By fitting with exponential function, the rising time is $0.97 \mathrm{~ms}$ at the control light power of 144 $\mathrm{mW}$ and the modulation frequency of $500 \mathrm{~Hz}$.

\section{All-optical actively Q-switched lasers}

The AOM, like acousto-optic and electro-optic modulator, can be applied into Q-switched laser generation as an active Q-switcher. In Fig. 5(a), a $980 \mathrm{~nm}$ laser diode is used as pump source to stimulate $1 \mathrm{~m}$ erbium-dopedfiber (YOFC, EDF80). The light is injected into cavity through a $980 / 1550 \mathrm{~nm}$ WDM. In order to protect the pump source, a polarization-insensitive isolators (ISO) guarantees the unidirectional light propagation. The laser is output through a $10 \%$ output OC. The AOM device modulates the transmittance of different wavelength to control output wavelength. The tunable filter (TF) provides a narrow spectral pass-band. A polarization controller (PC) is used to change intra-cavity polarization. The total length of cavity is about $22 \mathrm{~m}$.

Firstly, in order to achieve all-optical actively Qswitched laser, the pass band of TF must be smaller than the FSR of the AOM. The operation laser will possess high transmittance due to low loss and high $\mathrm{Q}$ factor in a

Table 1 | The current state-of-the-art all-optical modulator based on photothermal effect.

\begin{tabular}{|c|c|c|c|c|}
\hline Structure & Materials & Rising time (ms) & Falling time (ms) & Ref. \\
\hline $\mathrm{MZI}$ & Graphene & 9.1 & 3.2 & ref. ${ }^{14}$ \\
\hline $\mathrm{MZI}$ & MXene $\left(\mathrm{Ti}_{3} \mathrm{C}_{2} \mathrm{~T}_{x}\right)$ & 9.0 & 7.8 & ref. ${ }^{39}$ \\
\hline $\mathrm{MZI}$ & Phosphorene & 5.5 & 4.6 & ref. ${ }^{19}$ \\
\hline $\mathrm{MZI}$ & Boron nanosheets & 1.06 & 1.5 & ref. $^{33}$ \\
\hline $\mathrm{MZI}$ & $\mathrm{WS}_{2}$ & 7.3 & 3.5 & ref. $^{18}$ \\
\hline MI & Antimonene & 7.0 & 6.38 & ref. $^{40}$ \\
\hline MI & Bismuthene quantum dots & 3.42 & 3.37 & ref. $^{20}$ \\
\hline MI & MXene $\left(\mathrm{Ti}_{3} \mathrm{C}_{2} \mathrm{~T}_{x}\right)$ & 5.3 & 4.4 & ref. $^{22}$ \\
\hline MI & BQDs & 1.1 & 1.3 & This work \\
\hline MR & graphene & 0.3 & 0.21 & ref. $^{41}$ \\
\hline PR & $\mathrm{MoS}_{2}$-PVA & 0.55 & 0.77 & ref. $^{42}$ \\
\hline MRR & Phosphorene & $479 \mathrm{~ns}$ & $113 \mathrm{~ns}$ & ref. $^{21}$ \\
\hline MRR & Graphene & $556 \mathrm{~ns}$ & $1954 \mathrm{~ns}$ & ref. ${ }^{10}$ \\
\hline
\end{tabular}

MR: microfiber resonator; PR: polarization rotation; MRR: micro-ring resonator 

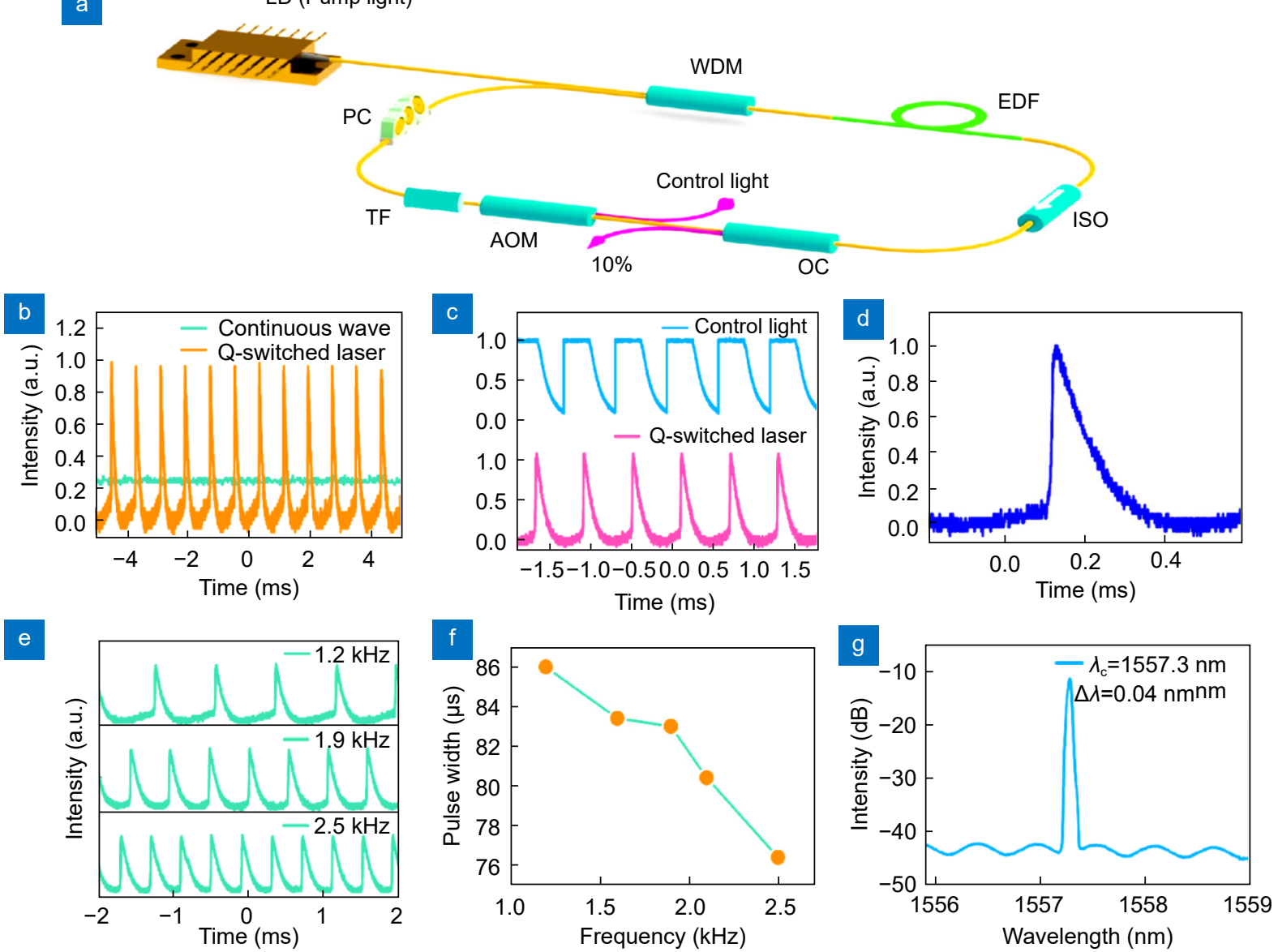

Fig. 5 | (a) The experimental diagram of the all-optical actively Q-switched lasers. (b) The state of continuous wave and Q-switched pulse laser. (c) The waveform of control light and pulse train. (d) Single pulse profile in (c). (e) The pulse trains under different modulation frequency. (f) The pulse width of Q-switched laser versus the modulation frequency. (g) The output spectrum of Q-switched laser.

fiber cavity, when the peak wavelength of the transmission curve of AOM is located near the pass-band of the TF. Otherwise, the high loss of intracavity will result in low power output. In the experimental, due to the FSR of $0.6 \mathrm{~nm}$, the pass-band of the TF is set to be $0.05 \mathrm{~nm}$. When guiding pump light of $50 \mathrm{~mW}$ into cavity, the laser cavity is operated in a continuous wave mode with the center wavelength of $1558 \mathrm{~nm}$ and the output power of $0.5 \mathrm{~mW}$ (green line in Fig. 5(b)). As the control light with the power of $130 \mathrm{~mW}$ and the modulation frequency of $1.2 \mathrm{kHz}$ is injected into the fiber cavity, a Qswitched pulse laser is obtained (orange line in Fig. 5(b)). Its repetition rate is equal to the modulation frequency of control light, illustrating an optically-controlled actively Q-switched fiber laser with controlled repetition rate is achieved. The operation stability of all-optical actively Qswitched fiber laser is mainly limited by the fiber-typed MI structure. The micro-nano devices such as micro-resonator or waveguide structure will improve the operation stability. Figure 5(c) and 5(d) gives the waveform of control light with the modulation frequency of $1.7 \mathrm{kHz}$, pulse train and single pulse profile (the pulse width of $83.4 \mu \mathrm{s})$. We can observe that the pulse possesses fast rising edge and slow falling edge, which results from the fast cavity oscillation and the reduction of photon density in the cavity. As the modulation frequency of control light increases, the repetition rate of Q-switched laser verities from $1.2 \mathrm{kHz}$ to $2.5 \mathrm{kHz}$ in Fig. 5(e), suggesting the actively Q-switched fiber laser can be controlled by the AOM. By optimizing laser cavity and using waveguide or micro-ring resonator structure to improve the response time of AOM, the repetition can be further scaled. Figure 5(f) shows the dependence of the pulse width on the modulation frequency. The shortest pulse width of Q-switched laser is $76 \mu$ s at the modulation frequency of $2.5 \mathrm{kHz}$. In addition, the output spectrum of the actively Q-switched pulses at the repetition rate of 2.5 $\mathrm{kHz}$ depicted in Fig. 5(g) with a 3-dB spectral width of $0.04 \mathrm{~nm}$ and the signal to noise ratio of $31 \mathrm{~dB}$ suggests that the Q-switched laser has excellent monochromaticity. 
It has tremendous potential in nonlinear frequency conversion and all-optical communication. The Q-switcher laser has the following advantages including broadband operation, large modulation depth, excellent monochromaticity, and easy implementation compared with other mechanism applied into all-optical actively Q-switched laser.

\section{Conclusions}

In conclusion, a BQDs-based all-optical modulator has been successfully fabricated to generate the all-optical actively Q-switched laser. The BQDs obtained from liquid-phase exfoliation was deposited onto the microfiber surface to interact with optical evanescent field. The BQDs-deposited microfiber exhibited efficient photothermal conversion as well as rapid photothermal response (photothermal heating curves of $385.4{ }^{\circ} \mathrm{C} / \mathrm{s}$ and $855.6{ }^{\circ} \mathrm{C} / \mathrm{s}$ at $50 \mathrm{~mW}$ and $100 \mathrm{~mW}$ ). The BQDs-based alloptical modulator achieved all-optical phase modulation and all-optical intensity modulation. The phase shift with a slope efficiency of $0.032 \pi / \mathrm{mW}$ and response time of $0.97 \mathrm{~ms}$ were superior to the similar device owing to the excellent thermal property of BQDs. Moreover, the output pulse laser achieved controllable repetition rate ranging from $1.2 \mathrm{kHz}$ to $2.5 \mathrm{kHz}$ utilizing the proposed all-optical modulator. This work demonstrates the advantageous optical properties of BQDs and its potential applications in optical communication and laser engineering for integrated optical switches and optical modulator devices. We hope this contribution may promote the experimental research of low-dimensional boron nanomaterials in future.

\section{References}

1. Huang HZ, Li JH, Deng J, GeY, Liu HG et al. Passively Qswitched Tm/Ho composite laser. Opto-Electron Adv 3, 190031 (2020).

2. Fang $\mathrm{YR}, \mathrm{Ge} Y Q$, Wang $\mathrm{C}$, Zhang $\mathrm{H}$. Mid - infrared photonics using 2D materials: status and challenges. Laser Photon Rev 4, 1900098 (2020).

3. Jiang $\mathrm{T}$, Yin $\mathrm{K}$, Wang $\mathrm{C}$, You J, Ouyang $\mathrm{H}$ et al. Ultrafast fiber lasers mode-locked by two-dimensional materials: review and prospect. Photonics Res 8, 78-90 (2020).

4. Hao $Q Q$, Wang C, Liu WX, Liu XQ, Liu J et al. Low-dimensional saturable absorbers for ultrafast photonics in solid-state bulk lasers: status and prospects. Nanophotonics 9, 2603-2639 (2020).

5. Tan T, Jiang XT, Wang C, Yao BC, Zhang H. 2D material optoelectronics for information functional device applications: status and challenges. Adv Sci 7, 2000058 (2020).

6. Liu HH, Yu Y, Song W, Jiang Q, Pang FF. Recent develop- ment of flat supercontinuum generation in specialty optical fibers. Opto-Electron Adv 2, 180020 (2019).

7. Nozaki K, Tanabe T, Shinya A, Matsuo S, Sato T et al. Subfemtojoule all-optical switching using a photonic-crystal nanocavity. Nat Photonics 4, 477-483 (2010).

8. Volz T, Reinhard A, Winger M, Badolato A, Hennessy KJ et al. Ultrafast all-optical switching by single photons. Nat Photonics 6, 605-609 (2012).

9. Yang S, Liu DC, Tan ZL, Liu K, Zhu ZH et al. CMOS-compatible $W_{2}$-based all-optical modulator. ACS Photonics $\mathbf{5}$, 342-346 (2018).

10. Qiu CY, Yang YX, Li C, Wang YF, Wu K et al. All-optical control of light on a graphene-on-silicon nitride chip using thermooptic effect. Sci Rep 7, 17046 (2017).

11. Ren $A B$, Feng $M$, Song $F$, Ren $Y Y$, Yang $S$ et al. Actively Qswitched ytterbium-doped fiber laser by an all-optical Q-switcher based on graphene saturable absorber. Opt Express 23, 21490-21496 (2015).

12. Williams RJ, Jovanovic N, Marshall GD, Withford MJ. All-optical, actively Q-switched fiber laser. Opt Express 18, 7714-7723 (2010).

13. Yun HG, Lee $\mathrm{SH}$, Lee $\mathrm{MH}$, Kim KH. An actively Q-switched single-longitudinal-mode fiber laser with an optically pumped saturable absorber. Laser Phys 23, 095107 (2013).

14. Ono M, Hata M, Tsunekawa M, Nozaki K, Sumikura H et al. UItrafast and energy-efficient all-optical switching with grapheneloaded deep-subwavelength plasmonic waveguides. Nat Photonics 14, 37-43 (2020).

15. Wang H, Yang NN, Chang LM, Zhou CB, Li SY et al. CMOScompatible all-optical modulator based on the saturable absorption of graphene. Photonics Res 8, 468-474 (2020).

16. Gan XT, Zhao CY, Wang YD, Mao D, Fang L et al. Grapheneassisted all-fiber phase shifter and switching. Optica 2, 468-471 (2015).

17. Yu SL, Wu XQ, Chen KR, Chen BG, Guo X et al. All-optical graphene modulator based on optical Kerr phase shift. Optica 3, 541-544 (2016).

18. Wu K, Guo CS, Wang H, Zhang XY, Wang J et al. All-optical phase shifter and switch near $1550 \mathrm{~nm}$ using tungsten disulfide $\left(\mathrm{WS}_{2}\right)$ deposited tapered fiber. Opt Express 25, 17639-17649 (2017).

19. Wang $Y Z$, Zhang F, Tang X, Chen X, Chen YX et al. All-optical phosphorene phase modulator with enhanced stability under ambient conditions. Laser Photon Rev 12, 1800016 (2018).

20. Wang $Y Z$, Huang WC, Zhao JL, Huang $H$, Wang $C$ et al. A bismuthene-based multifunctional all-optical phase and intensity modulator enabled by photothermal effect. J Mater Chem C 7, 871-878 (2019).

21. Cheng Z, Cao R, Guo J, Yao YH, Wei KK et al. Phosphoreneassisted silicon photonic modulator with fast response time. Nanophotonics 9, 1973-1979 (2020).

22. Wang $C$, Wang $Y Z$, Jiang $X T$, Xu JW, Huang WC et al. MXene $\mathrm{Ti}_{3} \mathrm{C}_{2} \mathrm{~T}_{x}$ : a promising photothermal conversion material and application in all - optical modulation and all - optical information loading. Adv Opt Mater 7, 1900060 (2019).

23. Mannix AJ, Zhang ZH, Guisinger NP, Yakobson BI, Hersam MC. Borophene as a prototype for synthetic $2 \mathrm{D}$ materials development. Nat Nanotechnol 13, 444-450 (2018).

24. Huang YF, Shirodkar SN, Yakobson BI. Two-dimensional Boron polymorphs for visible range plasmonics: a first- 
principles exploration. J Am Chem Soc 139, 17181-17185 (2017).

25. Zhou HB, Cai YQ, Zhang G, Zhang YW. Superior lattice thermal conductance of single-layer borophene. npj 2D Mater App/ 1, 14 (2017).

26. Xie ZJ, Meng XY, Li XN, Liang WY, Huang WC et al. Two-dimensional borophene: properties, fabrication, and promising applications. Research 2020, 2624617 (2020).

27. Vishnoi P, Pramoda K, Rao CNR. 2D elemental nanomaterials beyond graphene. ChemNanoMat 5, 1062-1091 (2019).

28. Jiang HR, Lu ZH, Wu MC, Ciucci F, Zhao TS. Borophene: a promising anode material offering high specific capacity and high rate capability for lithium-ion batteries. Nano Energy $\mathbf{2 3}$, 97-104 (2016).

29. Zhou BZ, Liu MJ, Wen YW, Li Y, Chen R. Atomic layer deposition for quantum dots based devices. Opto-Electron Adv 3, 190043 (2020).

30. Li HL, Jing L, Liu WW, Lin JJ, Tay RY et al. Scalable production of few-layer boron sheets by liquid-phase exfoliation and their superior supercapacitive performance. ACS Nano 12, 1262-1272 (2018)

31. Ranjan P, Sahu TK, Bhushan R, Yamijala SS, Late DJ et al. Freestanding borophene and its hybrids. Adv Mater 31, 1900353 (2019).

32. Feng BJ, Zhang J, Zhong Q, Li WB, Li S et al. Experimental realization of two-dimensional boron sheets. Nat Chem $\mathbf{8}$, 563-568 (2016).

33. Guo QB, Wu K, Shao ZP, Basore ET, Jiang P et al. Boron nanosheets for efficient all - optical modulation and logic operation. Adv Opt Mater 7, 1900322 (2019).

34. Bao QL, Zhang H, Wang Y, Ni ZH, Yan YL et al. Atomic - layer graphene as a saturable absorber for ultrafast pulsed lasers. Adv Funct Mater 19, 3077-3083 (2009).

35. Guo QB, Yao YH, Luo ZC, Qin ZP, Xie GQ et al. Universal near-infrared and mid-infrared optical modulation for ultrafast pulse generation enabled by colloidal plasmonic semiconductor nanocrystals. ACS Nano 10, 9463-9469 (2016).

36. Xie ZJ, Zhang F, Liang ZM, Fan TJ, Li ZJ et al. Revealing of the ultrafast third-order nonlinear optical response and enabled photonic application in two-dimensional tin sulfide. Photonics Res 7, 494-502 (2019).

37. Vega-Mayoral V, Vella D, Borzda T, Prijatelj M, Tempra I et al.
Exciton and charge carrier dynamics in few-layer $\mathrm{WS}_{2}$. Nanoscale 8, 5428-5434 (2016).

38. Guo J, Shi RC, Wang R, Wang YZ, Zhang F et al. Graphdiynepolymer nanocomposite as a broadband and robust saturable absorber for ultrafast photonics. Laser Photon Rev 14, 1900367 (2020).

39. Wu Q, Chen $S$, Wang $Y Z$, Wu LM, Jiang $X T$ et al. MZI-based all-optical modulator using $M X e n e \mathrm{Ti}_{3} \mathrm{C}_{2} \mathrm{~T}_{x}(\mathrm{~T}=\mathrm{F}, \mathrm{O}$, or $\mathrm{OH})$ deposited microfiber. Adv Mater Technol 4, 1800532 (2019).

40. Wang YZ, Huang WC, Wang C, Guo J, Zhang F et al. An alloptical, actively $\mathrm{Q}$-switched fiber laser by an antimonenebased optical modulator. Laser Photon Rev 13, 1800313 (2019).

41. Wang YD, Gan XT, Zhao CY, Fang L, Mao D et al. All-optical control of microfiber resonator by graphene's photothermal effect. Appl Phys Lett 108, 171905 (2016).

42. Wang YF, Wu K, Chen JP. All-optical modulator based on $\mathrm{MoS}_{2}$-PVA thin film. Chin Opt Lett 16, 020003 (2018).

\section{Acknowledgements}

We are grateful for financial supports from the State Key ResearchDevelopment Program of China (Grant No. 2019YFB2203503), National Natural Science Fund (Grant No. 61875138), The authors also acknowledge the support from the Instrumental Analysis Center of Shenzhen University (Xili Campus).

\section{Author contributions}

C. Wang and Q. Y. Chen contributed equally to this work. Y. P. Zhang and Y. N. Gong conceived the initial experiments on BQDs. Y. P. Zhang and H. Zhang supervised the project. Q. Y. Chen and Y. N. Gong synthesized and characterized BQDs samples; C. Wang, H. L. Chen, J. Liu and Y. Song conducted the experiments; J. Liu, D. L. Li and Y. Q. Ge participated and helped the data analysis. C. Wang and Q. Y. Chen co-wrote the manuscript with support from Y. N. Gong, Y. P. Zhang and H. Zhang. All authors contributed to the general discussion.

\section{Competing interests}

The authors declare no competing financial interests.

\section{Supplementary information}

Supplementary information for this paper is available at https://doi.org/10.29026/oea.2021.200032 